\title{
Die Auflagenpolitik des Internationalen Währungsfonds aus rechtlicher Sicht
}

\author{
Von Wolfgang Engshuber
}

\section{Einleitung}

Im Jahre 1944 wurden in Bretton Woods die beiden Schwesternorganisationen IWF und Weltbank gegründet, wobei die Aufgabenteilung den IWF als Währungsinstitution vorsah. ${ }^{1}$ In der Absicht, die destabilisierenden Wechselkursschwankungen der 30er Jahre, die durch einen Abwertungswettlauf gekennzeichnet waren, sowie die Unflexibilität der Wechselkurse unter dem Goldstandard zu vermeiden, vereinbarten die Gründungsmitglieder, ein System von Währungsparitäten mit Gold als gemeinsamen Maßstab zu errichten. Die internationalen Währungsbedingungen erwiesen sich jedoch als nicht statisch, und so mußten als Ergebnis weltwirtschaftlicher Entwicklungen einige der fundamentalsten Grundsätze des internationalen Währungssystems überprüft werden. Durch zwei Satzungsänderungen 1969 und 1978 paßte sich der Fonds den geänderten Verhältnissen an. Die letzte Änderung 1978 wurde durch den 1972 gegründeten Zwanzigerausschuß $\beta^{2}$ (Committee of Twenty) und die von ihm vorgelegte "Outline of Reform " ${ }^{3}$ ausgiebig vorbereitet und sollte zu einer Neuen Weltwirtschaftsordnung (NWWO) führen. Unter dem Druck der zweiten Olkrise erfolgte jedoch dann lediglich eine Änderung, die den gewandelten Verhältnissen Rechnung zollte (Schaffung der Sonderziehungsrechte, um den Reservebedarf zu decken [1969]; Úbergang zu flexiblen Wechselkursen [1978, de facto seit 1971]).

Gewandelt hat sich aber auch entscheidend die Funktion des IWF im internationalen Finanzsystem. Als im Jahre 1982 die drohende Zahlungsunfähigkeit Mexikos die Weltöffentlichkeit aufschreckte, wurde deutlich, daß die Verschuldungskrise nicht nur ein Problem für die verschuldeten Staaten darstellt, sondern das Weltwährungs- und -finanzsystem vor der Gefahr des Zusammenbruchs stand. Koordinierte Rettungsaktionen von Privatbanken, Zentralbanken, Regierungen, dem IWF und der Bank für Internationalen Zahlungsausgleich (BIZ) ermöglichten, die Zahlungsfähigkeit des Großschuldners wiederherzustellen. Deutlich wurde hierbei die Schlüsselrolle, die dem IWF bei der Lösung von Verschuldungskrisen zukommt.

Mittlerweile ist nahezu jede Umschuldung erst unter dem Gütesiegel des IWF möglich.

1 Siehe Articles of Agreement of the International Monetary Fund, Bretton Woods 22. 7. 1944, in Kraft getreten am 27. 12. 1945.

2 Gründung des Zwanzigerausschusses durch Resolution No. 27-10; 26. Juli 1972.

3 International Monetary Reform, Documents of the Committee of Twenty, Washington D.C. 1974. 
Der IWF verfügt wohl über die umfassendsten Informationen über die wirtschaftliche Lage seiner Mitglieder und besitzt zudem die Möglichkeit, auf seine Mitglieder Einfluß auszuüben. Gerade diese Einflußnahme stand seit jeher unter heftiger Kritik. ${ }^{4}$

Gegen die sogenannte Auflagenpolitik werden finanzpolitische, monetäre Argumente vorgebracht, die darauf hinzielen, andere makroökonomische Variablen zu beeinflussen. Die bisherige IWF-Politik ist bestrebt, interne Ursachen von Zahlungsbilanzungleichgewichten zu beseitigen sowie negative Auswirkungen externer Störungen auf den wirtschaftlichen Entwicklungsprozeß zu lindern: ${ }^{5}$

- Die Inflationsrate soll durch Verlangsamung des gesamten Geld- und Kreditwachstums, die Beschränkung der Kreditaufnahme des öffentlichen Sektors und der Auslandsverschuldung reduziert werden

- Abwertungen sollen überbewertete Währungen, die die Produktions- und die Konsumstruktur verzerren, auf eine reale Basis zurückführen

- Der Abbau von Handels- und Kapitalverkehrsbeschränkungen soll die Faktor- und Güterallokation verbessern

- Die Liberalisierung der Finanzmärkte zielt ab auf eine Erhöhung der inländischen Ersparnis und auf eine Ausweitung des nationalen Bankensystems.

Schlagzeilen von Hungeraufständen in Entwicklungsländern werden häufig mit dem IWF in Verbindung gebracht. Dies ist Ausgangspunkt für entwicklungspolitische Kritik am IWF. Die Bundestagsfraktion der Grünen richtete im Herbst '84 eine große Anfrage an die Bundesregierung über die Auflagenpolitik des IWF, in der sie u. a. folgende Beispiele aufführt: ${ }^{6}$

- IWF-Auflagen in Ägypten führten zur Streichung von Subventionen für Grundnahrungsmittel. Dies war Auslöser einer Revolte der Bevölkerung im Januar 1977 mit 79 Toten

- Einführung IWF-konformer Austeritätsmaßnahmen 1977 in Peru, verbunden mit Auseinandersetzungen zwischen Bevölkerung und Militär, die 20 Menschenleben und 300 Verhaftungen zur Folge hatten

- Auf Drängen des IWF im Mai 1978 erneuter Nahrungsmittel- und Transportkostensubventionsabbau mit Unruhen, die 30 Tote und 6000 Verhaftungen verursachten (Peru)

- Vom IWF erzwungener Reallohnabbau in Marokko mit der Folge eines Generalstreiks im Juni 1981 mit 66 (offiziell) bis 600 (inoffiziell) Toten, 2000 Verhaftungen und nachfolgendem Regierungsverbot für oppositionelle Zeitungen

- In Tunesien auf Initiative des IWF vorgenommene Subventionsstreichungen für Lebensmittel führten 1983 zu einer 100 \%igen Erhöhung der Preise für Hirse und Brot. Daraufhin kam es zur blutigen Niederschlagung eines Generalstreiks und als dessen Folge zum "Brotkrieg" mit mehreren hundert Toten.

4 Nowzard, Braham: The IMF and its Critics, New Jersey 1981.

5 Karsten, Ingo: Internationale Bankkredite an Entwicklungsländer, Baden-Baden 1984, S. 136 ff.

6 BT Drs. 10/1977, S. 10. 
Die Bundesregierung wies diese Behauptungen als willkürlich zurück und vertrat den Standpunkt, daß für hochverschuldete Staaten Anpassungsmaßnahmen unausweichlich seien und der IWF bei der Anpassung nur hilfreich beistehe. Ebenso verwies die Bundesregierung auf einen häufigen Vorwurf an die Entwicklungsländer, der Gang zum IWF sei viel zu spät erfolgt. Dies macht aber doch die Bestrebungen der Entwicklungsländer deutlich, den IWF mit seinen Auflagen zu meiden. Solange die internationalen Privatbanken Abnehmer für das zur Verfügung stehende Geld (Petrodollars) suchten, ${ }^{7}$ gelang eine Umgehung des IWF auch häufig. Inzwischen jedoch fließt kaum ein Privatkredit bzw. kommt keine Umschuldung zustande, bevor nicht ein Abkommen mit dem IWF erreicht wurde. Entwicklungsländer, die den IWF zu meiden versuchen, d. h. nicht um einen Kredit, der mit Auflagen verbunden ist, anrufen, machen geltend, ihre Interessen seien zu wenig berücksichtigt, sie hätten kaum ein Mitspracherecht und die Politik des IWF verletze sie in ihrem Selbstbestimmungsrecht. ${ }^{8}$

Die Berufung auf völkerrechtliche Grundsätze wird häufig seitens der Entwicklungsländer sowie der sie unterstützenden Literatur gebraucht, wenn auch bisweilen eine konkrete Herleitung oder Ausarbeitung fehlt. ${ }^{9}$ Ziel dieses Artikels soll es daher sein, ein bestimmtes Gebiet der Fonds-Tätigkeit, die Auflagenpolitik, unter rechtlichen Gesichtspunkten darzustellen und zu untersuchen, welchen Beitrag die Rechtswissenschaft zur Diskussion liefern kann.

\section{Rechtsquellen}

Primäre Rechtsquelle sind die Bestimmungen des IWF-Übereinkommens (Articles of Agreement) ${ }^{10}$ sie bilden in ihrer Gesamtheit vertragliches Währungsvölkerrecht. Das IWF-Übereinkommen stellt einerseits einen völkerrechtlichen Vertrag zur Gründung einer internationalen Organisation dar, enthält gleichzeitig aber auch die Verfassung dieser Rechtsgemeinschaft. Dem Gouverneurrat und dem Direktorium wird ausdrücklich keine legislative Kompetenz verliehen, obwohl die stark wechselnden wirtschaftlichen und politischen Verhältnisse es notwendig erscheinen ließen, die Zusammenarbeit der Mitgliedsstaaten mit dem Fonds diesen Verhältnissen jeweils anzupassen. Ursache hierfür mag gewesen sein, daß die Regierungsvertreter, die das IWF-Übereinkommen ausarbeiteten, nicht dazu bereit waren, das Exekutivdirektorium bzw. den Gouverneursrat zu "Eingriffen" in die Souveränität ihrer Staaten ausdrücklich zu autorisieren. ${ }^{11}$ Der Rat

7 Körner, Maaß, Siebold, Tetzlaff: Im Teuf elskreis der Verschuldung. Der Internationale Währungsf onds und die Dritte Welt, Hamburg 1984, S. 17.

8 Siehe etwa die Ausführungen des früheren Regierungsführers Jamaikas Manley, Michael: Jamaica, Struggel in the Periphery, London 1982.

9 Z. B. Nord-Süd-Kommission, Das Uberleben sichern, Köln 1980 sowie deren 2. Bericht, Hilfe in der Weltkrise, Köln 1983.

10 Ubereinkommen in der Fassung von 1976, abgedruckt in: BGB1. II, 78, S. 13 ff.

11 Hexner, Ervin P.: Das Verfassungs- und Rechtssystem des Internationalen Währungsfonds, 1960, S. 55 ff. 
der Gouverneure und das Direktorium haben jedoch die Kompetenz im Rahmen und im Funktionsbereich des IWF-Übereinkommens, allgemeine Verhaltensnormen für die Währungspolitik der Mitgliedsstaaten zu erlassen, die den Rahmen von technischen Ausführungsvorschriften überschreiten. Die Kompetenz zum Erlaß von Ausführungsvorschriften und Richtlinien bei der Inanspruchnahme von Fondsmitteln ergibt sich aus Art. V Abschnitt 3 IWF-Übereinkommen. Außerdem haben der Gouverneursrat bzw. das Exekutivdirektorium gemäß Art. XXIX IWF-Übereinkommen die Interpretationsbef ugnis bezüglich dieses Úbereinkommens.

\section{Zahlungsbilanzhilfen des IWF}

Der IWF stellt unter bestimmten Voraussetzungen Zahlungsbilanzhilfen zur Uberbrükkung temporärer Zahlungsbilanzungleichgewichte zur Verfügung (Art. I, V, VI IWFÚbereinkommen). Diese Zahlungsbilanzhilfen, die Möglichkeiten der Inanspruchnahme von Fondsmitteln, erscheinen, wenn man auf den wirtschaftlichen Zweck der Kreditbeschaffung abstellt, als Darlehnsvertrag. Der Fonds stellt dem Mitglied Finanzmittel in konvertierbarer Währung (meist US-\$) zur Verfügung, während das Mitglied seine, auf den Währungsmärkten nicht verwertbare, Währung hingibt.

Für die rechtliche Qualifizierung ist jedoch ausschließlich die rechtliche Gestaltung der Geschäfte maßgeblich. Das Fondsgeschäft stellt einen Vertrag dar, der sich aus einem Kauf von Fremdwährung gegen Zahlung von Eigenwährung und dem Rückerwerb von Eigenwährung durch den Kauf gegen Zahlung von konvertibler Währung oder Sonderziehungsrechten (SZR) zusammensetzt. Es handelt sich also um zwei Käufe, die durch die Setzung von Voraussetzungen miteinander zu einem Komplex verbunden sind. Kramer $^{12}$ qualifiziert wegen der Besonderheit des Vertragsgebildes die Fonds-Geschäfte als contractus sui generis. Im allgemeinen Sprachgebrauch werden die Fonds-Geschäfte als Ziehungen bezeichnet.

Die Zahlungsbilanzhilfen, die der Fonds seinen Mitgliedsländern zur Verfügung stellt, haben sich seit der Gründung des Fonds stetig entwickelt und der jeweiligen Weltwirtschaftslage angepaßt. Die Entscheidung, welche Möglichkeiten eingeräumt werden, trifft das Exekutivdirektorium. ${ }^{13}$ Der Umfang aller Kreditfazilitäten bestimmt sich prozentual zur jeweiligen Quote ${ }^{14}$ eines Mitgliedslandes.

Die ursprüngliche und immer noch bestimmende Möglichkeit ist die allgemeine Kreditfazilität, die sich untergliedert in verschiedene Tranchen. Innerhalb der sogenannten Reservetranche wird lediglich eine Ziehung auf die eigenen eingezahlten liquiden Reser-

12 Kramer, Jörg-Dietrich: Die Rechtsnatur der Geschäfte des Internationalen Währungsfonds, Berlin 1967, S. 62 ff. [104].

13 Eine zusammenfassende Darstellung enthält die Sammlung "Selected Decisions, International Monetary Fund, Washington DC", z. Z. 10. Ausgabe.

14 Die Quote eines Landes von Formeln abgeleitet, die etwa die Stellung eines Landes in der Weltwirtschaft widerspiegeln soll. 
ven des Fonds vorgenommen. Hierin liegt also de facto keine Inanspruchnahme von Fondsmitteln. Anträgen auf derartige Ziehungen kann nicht widersprochen werden. Darüber hinaus bestehen vier Kredittranchen, die jeweils $25 \%$ der Quote umfassen. Nach Ausschöpfung einer Tranche (z. B. 2. Tranche: $50 \%$ der Quote) hat die nächste Ziehung in der darüberliegenden Tranche (im Beispiel 3. Tranche) zu erfolgen. Die Bedingungen für eine Ziehung steigen entsprechend der Höhe der Tranche.

Zur Uberwindung gravierender Zahlungsbilanzungleichgewichte stellt der Fonds daneben die erweiterte Fondsfazilität für umfangreiche Hilfe zur Verfügung. Diese Fazilität wurde 1974 angesichts der deutlich werdenden Verschuldungssituation geschaffen. Voraussetzung für die Inanspruchnahme ist ein detaillierter Maßnahmenkatalog für die ersten und folgenden Zwölfmonatsperioden.

Neben diesen Möglichkeiten bestehen verschiedene Sonderfazilitäten, die an spezielle wirtschaftliche Situationen (Exporterlösausfälle, Kostenschwankungen bei Getreideimporten, Finanzierung von Rohstoffausgleichslagern, Olpreis) geknüpft sind. Diese Sonderfazilitäten sind jedoch zum Teil gänzlich ausgelaufen oder haben zumindest an Bedeutung verloren (vgl. Tabelle unter I.).

Die Politik des Fonds geht dahin, daß auch bei Krisen, die durch äußere Einflüsse bedingt sind, mehr und mehr eine strukturelle Anpassung erfolgen soll. Die Gründe hierfür sind zum einen, daß die äußeren Faktoren zu schon lange andauernden Zahlungsbilanzschwierigkeiten hinzukamen und zum anderen die Ursachen der steigenden Defizite, obwohl sie größtenteils externer Natur sind, keinen temporären Charakter haben. Mögen auch diese Gesichtspunkte zutreffen, so wird doch deutlich, daß die Anpassungslast verstärkt auf die hilfesuchenden Länder übertragen wird.*

\section{Bereitschaftskreditabkommen}

Voraussetzung für Ziehungen in den oberen Kredittranchen sowie der erweiterten Fondsfazilität ist ein Programm, in dem Ziele, Maßnahmen und Mittel dargelegt werden, die das Zahlungsbilanzungleichgewicht beheben sollen. Das IWF-Übereinkommen selbst sagt über das Programm wenig aus. Bedingung für die Inanspruchnahme ist gemäß Art. V Abschnitt 3 IWF-Übereinkommen, daß sie den Bestimmungen des IWFUbereinkommens und den hiernach beschlossenen Geschäftsgrundsätzen entspricht. Bereits $1952^{15}$ erfolgte durch Entscheidungen des Exekutivdirektoriums die Entwicklung von Bereitschaftskreditabkommen (stand-by arrangements). Ein Bereitschaftskreditabkommen ist eine Entscheidung des Fonds, mit welcher einem Mitglied versichert wird, während einer bestimmten Zeit und bis zu einem bestimmten Betrag Ziehungen in den oberen Kredittranchen zur Linderung seiner Zahlungsbilanzprobleme tätigen zu können. Die Zusage erfolgt, wenn das Mitglied ein Programm unterbreitet, das die Zustimmung

* S. Tabelle S. 26.

15 Horsefield, J. K.: The International Monetary Fund 1945-1965, Washington 1969, S. $230 \mathrm{f}$. 
$\approx \quad$ Mit geringen und hohen Auflagen verbundene Ziehungen, 1976-84 (in Milliarde

Am 30. April abs

$1976 \quad 1977 \quad 1978 \quad 1979$

I. Mit geringen Auflagen verbundene Ziehungen

Erste Kredeitranche

Olfazilität

Fazilität zur kompensierenden Finanzierung

Fazilität zur Finanzierung von Rohstoff-Ausgleichslagern

$\begin{array}{cccc}5,09 & 2,97 & 0,41 & 0,64 \\ 0,29 & 0,78 & 0,09 & 0,13 \\ 3,97 & 0,44 & - & - \\ 0,83 & 1,75 & 0,32 & 0,46 \\ { }^{1} & - & - & 0,05\end{array}$

II. Mit hohen Auflagen verbundene Ziehungen

$\begin{array}{llll}0,18 & 1,78 & 1,96 & 0,59\end{array}$

Kredittranche

$0,17 \quad 1,59 \quad 1,85 \quad 0,35$

Erweiterte Fondsfazilität

$0,01 \quad 0,19 \quad 0,11 \quad 0,24$

III. Insgesamt I + II

$5,27 \quad 4,75$

2,37

1,24

1 Weniger als 5 Mio. SZR.

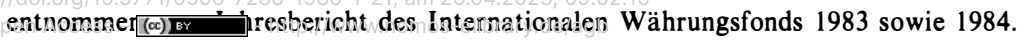

* Die Fazilität zur kompensierenden Finanzierung von Exporte Erlösausfällen, sowie von 
des Fonds findet. Bei der Inanspruchnahme der erweiterten Fondsfazilität werden erweiterte Vereinbarungen (extented Arrangements) abgeschlossen, welche, abgesehen von der längeren Laufzeit, keine Unterschiede zu den Bereitschaftskreditabkommen aufweisen. ${ }^{16}$

\section{Zustandekommen eines Bereitschaftskreditabkommens}

Ein Mitglied, das sich in Zahlungsbilanzschwierigkeiten befindet, tritt an den Fonds heran, um einen Kredit in einer bestimmten Höhe und Laufzeit zu erhalten. Der Fonds fordert nunmehr das Mitglied auf, anhand der von ihm festgelegten Richtlinien (Guidelines on Conditionality) ${ }^{17}$ ein Programm vorzulegen. Bei Ausarbeitung dieses Programms ist der Fonds auf Wunsch des Mitglieds behilflich. Findet das unterbreitete Programm die Zustimmung des Fonds, so entscheidet dieser durch die Gewährung eines Bereitschaftskreditabkommens über eine Kreditzusage in bestimmter Höhe und zu bestimmter Zeit. Ein Bereitschaftskreditabkommen besteht also aus zwei Dokumenten, dem Programm (letters of intent) und dem Bereitschaftskreditabkommen, der eigentlichen Entscheidung des Fonds.

\section{Rechtlicher Charakter}

Der rechtliche Charakter eines stand-by-arrangement hat praktische und politische Auswirkungen. Betrachtet man zunächst die Intentionen der Partner, so ist dem Fonds daran gelegen, daß die Verwendung der Mittel gemäß den durch die Satzung definierten Zielen des Fonds erfolgt. Zudem ist der Fonds bemüht, seine Ressourcen abzusichern. Das Mitglied, das die Hilfe des Fonds in Anspruch nehmen will, möchte seine wirtschaftlichen und finanziellen Ziele und Bestrebungen selbst bestimmen und vorantreiben. ${ }^{18}$

Gemäß diesen Vorgaben legt das Mitglied in seinem Programm (letter of intent) die Zielsetzung seiner Politik dar, während der Fonds in seiner Entscheidung festlegt, unter welchen Voraussetzungen das Mitglied Zahlungen erhält. Die zwei Dokumente sind aus juristischer Sicht keine internationalen Verträge, sondern Entscheidungen des Fonds bzw. des Mitglieds aufgrund einseitiger Absichtserklärung. ${ }^{19}$

16 Im folgenden wird auf Bereitschaftskreditabkommen eingegangen. Die Ausführungen gelten jedoch entsprechend auch für erweiterte Vereinbarungen.

17 Guidelines on Conditionality, Decision No 6056 - (79/38), Selected Decisions, 10. Ausgabe, 'S. $20 \mathrm{ff}$.

18 Gold, Joseph: The Legal Charakter of the Fund's stand-by Arrangements and Why it Matters, Washington 1980, Pamphlet Series No 35, S. 43.

19 Decision No 6056 - (79/38) Nr. 3; Selected Desisions, 10. Ausg. S. 20 ff. [21]; Gold, Joseph, The Stand-by Arrangements of the International Monetary Fund, Washington 1970, S. 46 f.; Roessler, Frieder, Pressures to adjust balance of payments disequilibria: an analysis of the power of the International Monetary Fund; International Organization, Vol XXX, 1976, S. 438; a.A.: Knieper, Rolf, Zurichtung nationaler Politik durch internationales Recht? Kritische Justiz 3/78, S. 277. 
Die Gründe, die gegen einen internationalen Vertrag sprechen, sind pragmatischer Natur:

a) Die Nichteinhaltung der Ziele des Programms, aus welchem Grund auch immer, soll keine Sanktionen auslösen

b) Es ist dadurch nicht notwendig, daß eine Eintragung gemäß Art. 102 UN-Charta und eine Veröffentlichung erfolgt (ob es zu einer Veröffentlichung kommt, hängt weitgehend vom Mitglied ab) ${ }^{20}$

c) Das Mitglied muß nicht das für Staatsverträge oft notwendige parlamentarische Verfahren durchführen

d) Der Fonds bindet sich nicht rechtsverbindlich. Dies ist insofern von Vorteil, als oftmals in Anbetracht der Laufzeit eine exakte Kriterienfestsetzung zu Beginn des Abkommens noch nicht möglich erscheint und zudem keine Flexibilität zuließe.

Die Möglichkeit eines Bereitschaftskreditabkommens ohne rechtliche Verbindlichkeit ist für den Fonds gegeben, da eine Auszahlung erst erfolgt, wenn das Mitglied Maßnahmen ergriffen hat. Auch durch die ratenweise Auszahlung kann jederzeit eine Beendigung des Abkommens erfolgen, wenn Bedingungen nicht eingehalten werden. Oftmals wird darauf hingewiesen, daß durch die rechtliche Würdigung von Bereitschaftskreditabkommen gesellschaftliche Gruppen, wie etwa Gewerkschaften, die von den Maßnahmen betroffen sind, keinerlei Möglichkeit haben, am Entscheidungsprozeß mitzuwirken, da kein parlamentarisches Verfahren notwendig ist und zudem keine Anfechtbarkeit besteht, da diese Gruppen keine Parteifähigkeit vor dem IGH besitzen. ${ }^{21}$ Diese Problematik kann jedoch schwerlich durch das Völkerrecht gelöst werden. Es bleibt dem politischen System eines jeden Mitglieds überlassen, wer die Entscheidungen trifft und wie gesellschaftliche Gruppen Einfluß nehmen können. Das vorgelegte Programm ist eine Entscheidung des Mitglieds für bestimmte Maßnahmen. Die politische Verantwortung trägt die Regierung bzw. das entscheidungsbefugte Organ.

Ein Bereitschaftskreditabkommen hat also rechtlich nur geringe Wirkung, um so entscheidender ist aber der Inhalt des Programms auf die wirtschaftliche Entwicklung. Dieser Inhalt wird offiziell natürlich vom Mitglied bestimmt, der Fonds erklärt sich jedoch nur zu einem Bereitschaftskreditabkommen bereit, wenn er inhaltlich mit dem Programm einverstanden ist. Die Ablehnungsbefugnis bedeutet in der Praxis eine große Einflußnahme. Es stellt sich nunmehr die Frage, ob dem Fonds bei dieser Tätigkeit, der Entscheidung, ob ein Programm bewilligt wird oder nicht, irgendwelche rechtlichen Schranken gesetzt sind.

20 Der Fonds veröffentlicht lediglich, welche Abkommen geschlossen wurden (im IMF Survey). Von Mitgliedern veröffentlichte Programme (letters of intent) siehe die Aufstellung bei Gold, Joseph, The Stand-by Arrangements of the International Monetary Fund, Washington 1970, Appendix cf. S. 269 f.; sowie bei Edwards, Richard, W. Jr., International Monetary Collaboration, New York 1985, S. $250 \mathrm{ff}$.

21 Gerster, Richard, Fallstricke der Verschuldung, Basel 1982, S. 125; Hoffmann, Diether, IWF Politik braucht soziale Komponente, in: Hans-Berthold Schäfer (Hrsg.), GefährdeteWeltfi nanzen, Bonn 1980, S. 241 ff. [244]; Körner, Maaß, Siebold, Tetzlaff, Im Teuf elskreis der Verschuldung. Der Internationale Währungsf onds und die Dritte Welt, Hamburg 1984, S. 226 ff. 


\section{Souveränität}

Internationale Organistionen üben in der heutigen Zeit eine die staatlichen Grenzen überschreitende, planende und verwaltende Tätigkeit aus und erfüllen viele Funktionen, die früher von den Einzelstaaten ausgeübt wurden oder die erst durch die zunehmende internationale Verflechtung entstanden; sie werden zu Trägern einer übernationalen Ordnung und Herrschaft, die sich auf Kosten der staatlichen Herrschaft entfaltet. Auszugehen ist jedoch davon, daß einzelne Staaten eine internationale Organisation gründen oder einer bestehenden beitreten und dabei freiwillig ohne jeglichen Zwang Teile ihrer bisherigen Entscheidungsbefugnis auf diese Organisation übertragen, um ein gemeinsames Ziel zu erreichen.

In Anbetracht der Weltwirtschaftssituation in den 30er Jahren bestand bei Gründung des IWF Einigkeit, die Entscheidungsbefugnis über Teile der Geld-, Währungs- und Wechselkurspolitik auf den IWF zu übertragen. Diesen Willen drückt auch jedes neue Mitglied durch seinen Beitritt aus. In welchen Bereichen der IWF die Entscheidungsbefugnis übertragen bekommen hat, ist dem IWF-Übereinkommen zu entnehmen.

Das IWF-Ubereinkommen enthält spezielle Regelungen ${ }^{22}$ sowie allgemeine Ziele und Richtlinien. ${ }^{23}$ Hinsichtlich der Bedingungen für die Inanspruchnahme der Fondsmittel verweist Art. V Abschnitt $3 I W F$-Übereinkommen auf eine notwendige Úbereinstimmung mit den Zielen des Übereinkommens. Dies sind gemäß Art. I i - ir IWF-Übereinkommen: internationale Zusammenarbeit auf dem Gebiet der Währungspolitik; Ausweitung und Wachstum des Welthandels und dadurch Förderung und Aufrechterhaltung eines hohen Beschäftigungsgrades und Realeinkommens; Stabilisierung der Währungen; Errichtung eines multilateralen Zahlungsverkehrs. Spezieller auf die Inanspruchnahme von Fondsmitteln eingehend:

- Art. I v IWF-Übereinkommen:

"... das Vertrauen der Mitglieder dadurch zu stärken, daß ihnen zeitweilig unter angemessenen Sicherungen die allgemeinen Fondsmittel zur Verfügung gestellt werden und ihnen so Gelegenheit gegeben wird, Unausgeglichenheiten in ihrer Zahlungsbilanz zu bereinigen, ohne zu Maßnahmen Zuflucht nehmen zu müssen, die dem nationalen oder internationalen Wohlstand schaden".

- Art. I vi IWF-Übereinkommen:

". . . in Ubereinstimmung mit Vorstehendem die Dauer der Ungleichgewichte der internationalen Zahlungsbilanz der Mitglieder abzukürzen und den Grad der Ungleichgewichte zu vermindern".

Die Satzung gibt also schon Anhaltspunkte, auf welche Faktoren die Auflagenpolitik einwirken kann. Art. V Abschnitt 3a IWF-Übereinkommen sieht vor, daß der Fonds Geschäftsgrundsätze bezüglich der Inanspruchnahme von Fondsmitteln beschließt. In den 70er Jahren ist die Auflagenpraxis wiederholt geändert worden. Gegen Ende des

22 Z. B. Art. IV, VIII Abschnitt 2, 3 IWF-Ubereinkommen.

23 Z. B. Art. I IWF-Ubereinkommen. 
Jahrzehnts empfand man die Notwendigkeit einer umfassenden Uberprüfung der Auflagen. ${ }^{24}$ Die Uberprüfung wurde vom Exekutivdirektorium 1978/79 vorgenommen und mit der Entscheidung No 6056 - $(79 / 38)^{25}$ abgeschlossen. Die Guideline on Conditionality beinhalten eine Reihe von Richtlinien für die Inanspruchnahme der Fondsmittel. Der Fonds ist grundsätzlich nicht befugt, auf landesspezifische, soziale oder politische Faktoren Einfluß zu nehmen. ${ }^{26}$ Oftmals wirken jedoch wirtschaftliche Auflagen auf diese Bereiche ein. Diesem Zusammenwirken hat der Fonds in Nr. 4 der Guidelines on Conditionality Rechnung getragen:

- "In helping members to devise adjustment programs, the Fund will pay due regard to the domestic social and political objectives, the economic priorities, and the circumstances of members, including the causes of their balances of payments problems".

Die Formulierung "due regard" gibt dem Fonds nicht die Macht, Einfluß zu nehmen, sondern es sollen vielmehr die Auswirkungen seiner wirtschaftlichen Maßnahmen berücksichtigt werden. ${ }^{27}$ Die Entscheidungsbefugnis über die landesspezifischen sozialen und politischen Faktoren soll also bei den Mitgliedern als Ausdruck ihrer Souveränität verbleiben. Nr. 9 der Guidelines on Conditionality legt fest, welche Erfüllungskriterien der Fonds setzen kann:

- "The number and content of performance criteria may vary because of the diversity of problems and institutional arrangements of members. Performance criteria will be limited to those that are necessary to evaluate implementation of the program with a. view to ensuring the achievement of its objectives. Performance criteria will normally be confined to (i) macroeconomic variables, and (ii) those necessary to implement specific provisions of the Articles or policies adopted under them. Performance criteria may relate to other variables only in exceptional cases when they are essential for the effectiveness of the members' program because of their macroeconomic impact." Schwierigkeiten in der Praxis bereitet die Definition von "macroeconomic variables". Eine allgemein anwendbare Abgrenzung von makroökonomischen Kriterien und Kriterien, die in die Mikroökonomie eingreifen und somit, da vom IWF-Übereinkommen nicht gedeckt, eine Souveränitätsverletzung darstellen, ist schwer zu finden. ${ }^{28}$ Eine Entscheidung ist wohl jeweils am Einzelfall zu treffen. Gerade wegen dieser Schwierigkeiten sollte der Fonds bemüht sein, durch eine kontinuierliche Praxis hier Klarheit zu schaffen.

24 Guitian Manuel, Die Auflagen des IWF und der internationale Anpassungsprozeß: die wechselhaften 70er Jahre; Finanzierung und Entwicklung, März 1981, S. 11.

25 Decision No 6056 - (79/38); Selected Decisions, 10. Ausg., S. $20 \mathrm{ff}$.

26 Gold, Joseph, Political Considerations are Prohibited by Articles of Agreement when the Fund Considers Requests of Use of Ressources, IMF-Survey 1983, S. 146.

27 Gold, Joseph, Political Considerations are Prohibited by Articles of Agreement when the Fund Considers Requests for Use of Ressources, IMF-Survey 1983, S. 146; Grosche, Günther, Zur Finanzierung der Leistungsbilanzdefizite der Nicht-Öl-Entwicklungsländer, in: Nationale Entwicklung und internationale Zusammenarbeit, Berlin/Heidelberg 1983, S. 272 ff. [279].

28 Momentan wird im Fonds und in der Weltbank diskutiert, wie die beiden Organisationen im Hinblick auf den eigenständigen Charakter und getrennten Aufgabenbereich auf die geänderten Verhältnisse (andauernde Krisen) reagieren sollen. 
Nachdem in den 70er Jahren versucht worden war, durch Sonderfazilitäten, insbesondere der Olfazilität, auf die Zahlungsbilanzstabilität einzuwirken, setzte sich zu Beginn der 80er Jahre mehr und mehr der Trend zu Strukturveränderungen durch. ${ }^{29}$ Ziel sind Veränderungen in den Volkswirtschaften, eine strukturelle Änderung in Produktion und Nachfrage. Die Wiedererlangung einer haltbaren Zahlungsbilanzposition bei Preisstabilität und angemessenem Wachstum ohne Rückgriff auf Maßnahmen, die die Freiheit des Handels- und Zahlungsverkehrs einschränken, ist Hauptziel des Fonds. Nachdem zunächst zur Erreichung dieser Ziele Maßnahmen der Nachfragesteuerung insbesondere im Finanz- und Kreditbereich ergriffen wurden, wird nunmehr verstärkt auf die Angebotssteuerung Wert gelegt. Eine optimale Ausnutzung des Angebotspotentials ist aber schon immer das letztliche Ziel gewesen. Oftmals wirken Maßnahmen sowohl auf die Nachfrage- als auch auf die Angebotsseite ein. Die Beziehung zwischen Angebots- und Nachfragesteuerung wird durch verschiedene Maßnahmen illustriert, die in der Regel einen Stabilisierungsplan bestimmen. ${ }^{30}$ Dies sind: die Preis-, Steuer- und Subventionspolitik des öffentlichen Sektors, die dazu beitragen kann, finanzielle Ungleichgewichte zu beseitigen und die Effizienz im öffentlichen Bereich zu fördern; die Zinspolitik zur Erhöhung der inländischen Ersparnisse und zur Verbesserung der Ressourcenallokation; die Wechselkurspolitik, die hilft, die Absorption und die außenwirtschaftliche Flanke zu kontrollieren, die Einkommenspolitik, die die Ansprüche im Rahmen der verfügbaren Ressourcen hält; die Festsetzung von Kreditobergrenzen. Maßnahmen in diesen Bereichen sind für die Ziele des Fonds entscheidend, weil sie Ersparnis und Investition stärken, Faktoren, die Basis für die Angebotsausweitung und für die gesunde Entwicklung einer Volkswirtschaft sind.

Der Fonds ist häufig dem Vorwurf ausgesetzt, seine Auflagenpolitik treffe die breite Bevölkerung am schwersten (Erhöhung der Lebensmittelpreise), während andere Gebiete, wie etwa der Rüstungsetat nicht angetastet werden. ${ }^{31}$ Es wird gefordert, der Fonds solle in seinen Auflagen unter anderem auf eine gerechtere Einkommensverteilung hinwirken. ${ }^{32}$ Demgegenüber macht der Fonds geltend, daß es nicht in seiner Entscheidungsmacht liegt, auf soziale und politische Beschlüsse von souveränen Mitgliedern Einfluß zu nehmen..$^{33}$ Die Frage ist jedoch, ob man durch Interpretation des Art. I IWF-Übereinkommen nicht eine derartige Einflußnahme rechtfertigen könnte. ${ }^{34}$ In Betracht kommt:

29 Gute Ubersicht: Auf satzreihe von Guitian, Manuel, Der IWF und der internationale Anpassungsprozeß; Finanzierung und Entwicklung, Dezember 1980, März 1981, Juni 1981.

30 Guitian, Manuel, Die Auflagen des IWF und der internationale Anpassungsprozeß, ein Blick auf die 80er Jahre. Finanzierung und Entwicklung Juni 1981, S. 16.

31 Am gravierendsten ist wohl folgendes Beispiel: Indien erhielt im Jahre 1981 eine Kreditzusage von 5,8 Milliarden \$. Kurz danach kaufte es für 3,3 Mrd. \$ Kampfflugzeuge vom Typ Mirage 2000 von Frankreich. Frankreich hatte die Kreditzusage stark unterstützt, während die USA dagegenstimmten (Wall Street Journal 23. 11. 81, S. 26).

32 Bundestagsanfrage der Grünen, BT Drs. 10/1977, S. 20.

33 Larosière, de J., Does the Fund Impose Austerity? Washington, Pamphlet, Juni 1984.

$34 \mathrm{Zu}$ den Interpretationsmöglichkeiten und Methoden siehe: Gold, Joseph, Interpretation by the International Monetary Fund of its Articles of Agreement I, II, in: International and Comparative Law Quarterly 1954, S. 256-276 bzw. 1967 , S. 289-329. 
a) Art I ii IWF-Úbereinkommen

"... die Ausweitung und ein ausgewogenes Wachstum des Welthandels zu erleichtern und dadurch zur Förderung und Aufrechterhaltung eines hohen Beschäftigungsgrades und Realeinkommens sowie zur Entwicklung des Produktionspotentials aller Mitglieder als oberste Ziele der Wirtschaftspolitik beizutragen"

Hier wird durch die Formulierung "und dadurch" deutlich, daß Primärziel die Förderung des Welthandels ist und sich dementsprechend die Maßnahmen hierauf zu erstrecken haben. Als gewünschte Folge sollen das Realeinkommen und der Beschäftigungsgrad gesichert werden.

b) Art. I v IWF-Übereinkommen

"Die Unterstützung soll dazu dienen, um die Zahlungsbilanz auszugleichen . . ., ohne zu Maßnahmen Zuflucht nehmen zu müssen, die dem nationalen oder internationalen Wohlstand schaden."

Der Begriff "nationaler Wohlstand" ist jedoch auslegungsfähig, und es bleibt dem einzelnen Mitglied weitgehend überlassen, was es darunter versteht. So kann geltend gemacht werden, daß eine zu geringe Verteidigungskraft den nationalen Wohlstand mehr gefährdet als höhere Lebensmittelpreise. Es bleibt somit festzuhalten, daß das IWF-Übereinkommen zumindest keine Verpflichtung des Fonds enthält, den Mitgliedsländern eine "gerechte" Sozialpolitik vorzuschreiben.

Betrachtet man die dargestellten Einflußnahmemöglichkeiten des Fonds, so wird deutlich, daß die Souveränität, das Recht eines jeden Staates, frei sein politisches, soziales, wirtschaftliches und kulturelles System zu wählen und zu entfalten, beträchtlich tangiert wird. Zwar erfolgt der Beitritt zum IWF freiwillig, und auch der Abschluß eines Bereitschaftsabkommens kommt nur bei einem Kreditwunsch des Landes zustande, aber in der Realität hat ein überschuldetes Land kaum eine Möglichkeit, ohne eine Vereinbarung mit dem Fonds seine Schwierigkeiten zu lösen. Die gesamten Umschuldungsprogramme, an denen einzelne Staaten sowie die Privatbanken beteiligt sind, kommen in der Regel erst zustande, nachdem mit dem IWF ein Abkommen geschlossen wurde. Der Hinweis auf die "Freiwilligkeit" der Inanspruchnahme von Fondsmitteln ist daher zu formalistisch, vielmehr ist das Fondshandeln unter dem Gesichtspunkt der Vereinbarkeit mit dem Grundrecht der Staaten, der Souveränität, zu beurteilen. Es ist am Einzelfall herauszuarbeiten, inwieweit die geforderten Maßnahmen die freie Entscheidung eines Staates, über sein politisches, soziales und wirtschaftliches System zu bestimmen, einschränkt. 


\section{Gleichheit der Staaten}

Der völkerrechtliche Gleichheitssatz hat im IWF-Übereinkommen seinen Niederschlag gefunden in dem Prinzip der Gleichbehandlung (uniformity). ${ }^{35}$ Dieses Prinzip besteht aus zwei Elementen. Zum einen schafft das IWF-Úbereinkommen die gleichen Rechte und Pflichten für alle Mitgliedsländer, zum anderen findet die Fondspolitik gleiche Anwendung für alle Mitglieder. Es sollen keine Vor- oder Nachteile aus der finanziellen Stärke oder Schwäche oder aufgrund anderer Charakteristika entstehen. Eine Diskriminierung soll vermieden werden. Gleichheit wird im Fonds nicht als formelle, auch wenn es solche Anwendungsbereiche gibt, sondern als materielle Gleichheit verstanden. Auf diese Weise erhält die Quote eines Mitglieds entscheidendes Gewicht. Von grundsätzlicher Bedeutung ist das Prinzip, wenn es um die Diskussion etwaiger Vorrechte für Entwicklungsländer geht. Bereits bei der Entstehung des Abkommens in Bretton Woods war zunächst in Art. I ii IWF-Übereinkommen der Begriff "underdeveloped countries" vorgesehen, er wurde jedoch dann ersetzt durch "to all countries, with due regard to the needs of economically backward countries «. ${ }^{36}$ Bei der Ausarbeitung der Reformvorschläge zur zweiten Satzungsänderung ${ }^{37}$ tauchte wiederholt die Frage auf, ob spezielle Rechte für Entwicklungsländer eingefügt werden sollen. ${ }^{38}$ Eine explizite Nennung von Entwicklungsländern ist jedoch dann unterblieben, um den Gleichbehandlungsgrundsatz zu wahren. So kommen zwar in der Praxis einige Fazilitäten derzeit wohl nur für Entwicklungsländer in Frage, eine ausdrückliche Differenzierung wurde jedoch nicht getroffen. Als der Fonds bemüht war, die Erlöse von Goldverkäufen teilweise den Entwicklungsländern zur Verfügung zu stellen, sah er sich dem Widerspruch mit dem Prinzip der Gleichbehandlung konfrontiert. Es wurde aus diesem Grunde die Lösung über die Errichtung eines Trustfonds ${ }^{39}$ der nicht unmittelbar dem IWF unterstellt ist, gewählt.

Wendet man nun das Prinzip auf die Mittelvergabe des Fonds an, so folgt daraus, daß jedes Mitglied unter gleichen Voraussetzungen und zu gleichen Bedingungen Zugang zu den Fondsmitteln haben muß. Das Problem ist aber, wann gleiche Voraussetzungen bzw. gleiche Bedingungen vorliegen. Hier ist der Ansatzpunkt für viele Kritiker des Fonds, die geltend machen, der Fonds unterstütze einseitig kapitalistische oder autoritäre Systeme. ${ }^{40}$

35 Gold, Joseph, Uniformity as a legal principle of the International Monetary Fund, Law and Policy in international business 1975 , S. $765 \mathrm{ff}$.

36 Nachweise bei Gold, Joseph: Uniformity as a legal principle of the International Monetary Fund, Law and Policy, in: international business, 1975, S. $765 \mathrm{ff} .774 \mathrm{f}$.

37 "Outline of Reform" International Monetary Reform, Documents of the Dommittee of Twenty, 1974.

38 Gold, Joseph, The IWF and Developing Countries: Median Journal of international Law, 1981, S. $497 \mathrm{ff}$.

39 Gold, Joseph, Trust fonds in international law. The contribution of the IMF to a code of principles; A.J.I.L. 72 (1978), S. $856 \mathrm{ff}$.

40 Vgl. Anfrage der Grünen im Bundestag, BT Drs. 10, 1977, S. 11 ff.; Körner, Maaß, Siebold, Tetzlaff, Im Teufelskreis der Verschuldung. Der internationale Währungsfonds und die Dritte Welt, Hamburg 1984, S. 83 ff. m. w. N. 
Bereits unter dem Gesichtspunkt der Souveränität ${ }^{41}$ wurde kurz erläutert, welche Erfüllungskriterien der Fonds setzen kann. Offen geblieben ist jedoch die Frage nach der Strenge der Auflagen im Verhältnis zur Situation des kreditsuchenden Landes. Der Fonds hat hierzu "unwritten guidelines" herausgearbeitet. Diese beschreiben genauer die Faktoren, die zu berücksichtigen sind, wenn die Situation eines Landes und der Kreditrahmen bzw. die Strenge der Auflagen erörtert werden. Die "unwritten guidelines" nennen als maßgebliche Faktoren:

1. Höhe des Zahlungsbilanzdefizits im Verhältnis zur Quote und dem beantragten Zahlungsbedarf seitens des Fonds, unter Berücksichtigung anderweitiger Finanzquellen und dem Begehren einen annehmbaren Stand der Währungsreserven aufrechtzuerhalten.

2. Natur des Zahlungsbilanzdefizits: strukturell oder zyklisch, und die damit verknüpfte Frage nach der Anpassungsdauer.

3. Qualität und Strenge des Anpassungsprogramms einschließlich der Angemessenheit der Politik sowie der Zeitpunkt des Gesuchs.

4. Beurteilung der bisherigen Zusammenarbeit bei Inanspruchnahme von Fondsmitteln und die Erfolgsaussichten, einen dauerhaften Gebrauch dieser Mittel zu vermeiden.

Bei Ziehungen in den oberen Kredittranchen wird auf jeden Fall ein umfassendes Anpassungsprogramm mit einschneidenden Maßnahmen zu Beginn und mit der Aussicht erwartet, daß die Anpassung grundsätzlich abgeschlossen ist, bevor die Rückzahlung beginnt.

Der Fonds hat somit einige Kriterien festgelegt, die für seine Entscheidung ausschlaggebend sind. Anhand dieser Kriterien ist ein Vergleich von verschiedenen Kreditanträgen möglich und trotz der Schwierigkeiten, bei der Bewertung von wirtschaftlichen Faktoren sollte es realisierbar sein, eine Verletzung des Gleichbehandlungsgrundsatzes festzustellen. ${ }^{42}$

\section{Begrenzter Aufgabenbereich und Wirkungskreis}

Trotz einer gewissen Deckung mit den vorgenannten Grenzen des Wirkungskreises des IWF's, läß sich jedoch noch eine weitere Grenze herausstellen. Wie bereits angedeutet, wird die Entscheidungsfindung im IWF nach dem Prinzip des "weighted voting «43 durchgeführt. Bei jeglicher Art von Stimmengewichtung ist zu untersuchen, nach welchen Kriterien und Faktoren die Stimmen gewichtet werden. Im IWF erfolgt dies anhand der den Ländern zugeteilten Quote. Diese Quote wird von Formeln abgeleitet, die in

41 Siehe oben unter III.

42 Die Problematik ist ähnlich der Uberprüfung von Ermessensentscheidungen anhand des Gleichheitssatzes im innerstaatlichen Recht.

43 Eingehende Darstellung: Gold Joseph, Voting and Decisions in the International Monetary Fund, Washington D.C. 1972. 
etwa die relative Position eines jeden Landes innerhalb der Weltwirtschaft widerspiegeln. Zur Zeit wird eine Kombination von fünf Formeln verwandt. ${ }^{44}$

Findet in einer internationalen Organisation eine Stimmengewichtung statt, so ist dies mit dem völkerrechtlichen Gleichheitssatz nur vereinbar, wenn ein direkter Zusammenhang zwischen Gewichtungskriterium und Aufgaben- bzw. Wirkungskreis besteht. Es findet in diesem Fall keine formelle, sondern eine materielle Gleichheit Anwendung. Dies bedeutet auf den IWF bezogen, daß aus dem Prinzip des "weighted voting " und dem Abstellen auf wirtschaftliche Größen folgt, daß der Aufgaben und Wirkungskreis nur wirtschaftlicher Art sein darf. ${ }^{45}$

Eine Betrachtung des IWF ergibt, daß alle Aufgaben und Befugnisse, die das Abkommen vorsieht sich auf Wirtschafts- und Währungsgebiet erstrecken. Auch die Entscheidungen, Grundsätze sowie Interpretationen durch den Gouverneursrat, bzw. das Exekutivdirektorium lassen nur wirtschaftliche Gesichtspunkte erkennen. ${ }^{46}$

Kritiker $^{47}$ machen jedoch geltend, daß oftmals andere als wirtschaftliche Faktoren die Entscheidungen des IWF prägen. Zu diesem Ergebnis kommt auch eine Studie, die von Caleb Rossiter ${ }^{48} 1983$ in den USA erstellt wurde. Hierin wird dargestellt, daß für die Entscheidung des amerikanischen Exekutivdirektors wiederholt andere als wirtschaftliche Faktoren ausschlaggebend waren. So soll es eine inoffizielle "Hit-List" geben, auf der Staaten genannt sind, die keine IWF-Unterstützung erhalten sollen. Auf Grund eines Stimmenanteils von ca. $20 \%$ haben die USA erheblichen Einfluß auf die Entscheidungen des IWF. Sind nun aber für die USA oder eine andere Staatengruppe politische Erwägungen maßgeblich, so handelt der Fonds immerhin mit diesem Stimmenanteil politisch und dies kann oftmals ausschlaggebend sein.

Ein weiteres Beispiel für derartig "sachfremde Erwägungen" ist das Verhalten einiger arabischer Staaten gegenüber Kreditgesuchen afrikanischer Staaten, die mit Israel diplomatische Beziehungen pflegen.

1982 sorgte der Fall "Südafrika" für Schlagzeilen. Südafrika war mit einem Kreditwunsch an den Fonds herangetreten, worauf sich Protest anderer afrikanischer Staaten breitmachte. Die Generalversammlung der Vereinten Nationen entschied mit 123:3 Stimmen ${ }^{49}$ für eine Empfehlung an den IWF, es solle keine Kreditzusage erteilt werden. Die ablehnenden Industrieländer vertraten die Auffassung, politische Erwägungen (Ablehnung der Apartheitspolitik) sollten aus dem Entscheidungsprozeß des IWF herausgehalten werden und unter wirtschaftlichen Gesichtspunkten sei die Kreditgewährung

44 IWF Jahresbericht 1983 S. 20/21.

45 Barents, R. in Kapteyn, Lawwaars, Kooijmans, Schermers, Boomkamp: International Organization and Interpretation Vol IB, 1982 Dir.I.B.1.16.

46 Selected Decisions, 10. Ausgabe 1983.

47 Bundestagsanfrage der Grünen, BT Drs. 10/1977 S. $10 \mathrm{ff}$.

48 Caleb Rossiter, The Financial Hit List, Center for International Policy, Washington D.C. 1984.

49 Die Gegenstimmen stammen von den USA, Großbritannien, Bundesrepublik Deutschland (Wall Street Journal 22. 10. 82 S. 1). 
zu befürworten. Nach langer Diskussion ${ }^{50}$ wurde Südafrika der Kredit zugesprochen. Die Entscheidung wurde, wie im Fonds allgemein üblich ohne formelle Abstimmung (Konsensprinzip) gefällt. Bemerkenswert ist aber auch die weitere Entwicklung. Im .Gesetz vom 30. 11. 1983, ${ }^{51}$ mit dem die USA der achten Quotenerhöhung zustimmen, wird der amerikanische Exekutivdirektor angewiesen, gegen jeglichen Kredit an Südafrika oder ein kommunistisches Land zu stimmen, es sei denn er erhält eine andere Weisung. Diese Einflußnahme der USA wurde innerhalb des Landes durch die Demokraten verurteilt, das Gesetz fand jedoch eine ausreichende Mehrheit. ${ }^{52}$

Eine Kontrolle über die Einflußnahme auf die Entscheidungen des Fonds ist in der Praxis sicher sehr schwierig, da kein Exekutivdirektor gehalten ist, seine Entscheidungsgründe zu nennen. Fälle wie die Anweisung an den amerikanischen Direktor werden meist nicht publiziert. Zudem verwenden die Exekutivdirektoren in ihren Stellungnahmen nur wirtschaftliche Argumente. Trotz dieser Schwierigkeiten stellt es eine wichtige Aufgabe des Fonds dar, Einflüsse nicht wirtschaftlicher Art abzuwehren, damit nicht seine Neutralität und seine Aufgabe im Weltwährungssystem ernstlich in Frage gestellt ist.

Der einzige Schutz vor Fremdbeeinflussung bei der Mittelvergabe ist momentan darin zu sehen, daß für den Abschluß eines Bereitschaftskreditabkommen nur eine einfache Stimmenmehrheit erforderlich ist. ${ }^{53}$ Auf diese Weise wird vermieden, daß einzelne Staaten (USA) oder Staatengruppen eine Sperrminorität haben und sich damit ihr Einfluß verstärkt. $^{54}$

\section{Resümee}

Zum Beitrag, den die Rechtswissenschaft zur Diskussion über die Auflagenpolitik des IWF leisten kann, ist festzustellen, daß das Völkerrecht deutliche Grenzen der Einflußnahme auf die einzelnen Mitglieder setzt. Diese Grenzen sind bisher kaum herausgestellt worden. Sie sind von Bedeutung für den Fonds, die Mitgliedstaaten und auch für die gesellschaftlichen Gruppen bzw. die Bevölkerung in den Mitgliedsländern.

50 Wall Street Journal 4. 11. 1982 S. 5 (Wall Street Journal 5. 5. 1983, S. 1; 26).

51 P.L. 98-181 Supplemental Appropriations Act, 1984; Domesting Housing and International Recovery and Financial Stability Act; Title VIII, International Monetary Fund 22 USC 286aa.

52 Washington Post, 19. 8. 83, A22; 6. 10. 83; C15; 7. 10.83, A22.

53 Gold Joseph, Political Considerations Are Prohibited by Articles of Agreement when the Fund Considers Requests for the Use of Ressources IMF Survey 1983, S. 147.

54 Grenada erhielt z. B. Ende August 1983 einen 14,3 Mill US Kredit obwohl es aufder "Hit List " stand und die USA auch Widerstand leisteten. (Wall Street Journal 26. 8. 1983 S. 18, Wall Street Journal 16. 11. 1983 S. 21). 


\section{1. $I W F$}

Dem Fonds sollte daran gelegen sein, seine Grenzen klar herauszustellen um nicht die "Prügelknabenrolle" für unpopuläre Maßnahmen übertragen zu bekommen. Welche gesellschaftliche Gruppen die Auswirkungen von Sparmaßnahmen zu tragen haben (z. B. Kürzungen bei den Lebensmittelsubventionen, Steuererhöhungen, oder Einsparungen bei den Militärausgaben) ist eine Entscheidung der jeweiligen Regierung und von ihr auch politisch zu verantworten.

Desweiteren ist eine Kenntlichmachung des begrenzten Aufgaben- und Wirkungskreises wichtig, um sich vor Fremdbeeinflussung schützen zu können.

\section{Mitgliedsstaaten}

Jedem kreditsuchenden Land sollten die Grenzen der Einflußnahme durch den Fonds bewußt sein. Der Fonds ist nicht befugt, mikroökonomische Erfüllungskriterien zu setzen. Auch andere als wirtschaftliche Kriterien sind unzulässig. Zudem ist der Fonds an den Grundsatz der Gleichbehandlung gebunden, der die Mitglieder vor Diskriminierung schützt. Bei Verletzung dieser Grenzen müßte ein Mitglied in den ansonsten geheimen Verhandlungen mit dem IWF-Stab an die Öfentlichkeit treten und die Frage etwa dem Exekutivdirektorium vorlegen.

\section{Gesellschaftliche Gruppen}

Die dargelegten Grenzen des Fondshandeln machen deutlich, daß die politische Verantwortung zum Großteil bei den Regierungen liegt. Dies muß den gesellschaftlichen Gruppen (etwa Gewerkschaften) deutlich sein, da sie auf den IWF keinen Einfluß nehmen können und sich somit of tmals machtlos fühlen. Die Regierungen sollen sich nicht durch Verweisung auf den IWF ihrer politischen Verantwortung entziehen können.' 


\title{
ABSTRACTS
}

\section{Existing Law and the Implementation of a Bill of Rights: A Caribbean Perspective}

\author{
By Margaret DeMerieux
}

The introduction of constitutionally guaranteed fundamental rights and freedoms into an existing system of laws, requires the law-maker to take account of the impact of these laws on the new right and vice versa. A decision to preserve the validity of existing laws, effectively makes of them a set of limitations defining the extent of the new rights. In the Commonwealth Caribbean, some states have used the device of a savings clause to validate existing law against new rights. In other states the absence of a savings clause allows courts to pronounce existing laws invalid as infringing the new rights. In either case however existing law forms the background to the interpretation and understanding of the new regime, with implications for its effectiveness or otherwise. The article examines some of the problems raised in the Caribbean, in this area.

\section{The IMF's Policy On Conditionality: A Legal Perspective}

\section{By Wolfgang Engshuber}

When the IMF was founded at Bretton Woods in 1944, it was intended that this organisation should prevent global monetary crises such as those of the 1930s. Meanwhile the IMF has come to play a major part in the attempts to control the international debt crisis. Nearly every rescheduling of debt now requires a stand-by or extended arrangement with the IMF. These arrangements between the debtor nation and the IMF are a crucial issue in the discussions about a New International Economic Order.

Developing countries accuse the IMF of violating the principles of sovereignty and the equality of states.

The article describes the legal framework of the IMF's conditionality policy and points out the limits of the IMF's powers. Even the institution's own "Guidelines on Conditionality" accept that the Fund has to pay due regard to the domestic social and political objectives and the circumstances of members.

A clarification of the limited powers of the IMF is important for all interested parties. If the IMF should inappropriately pre-empt domestic political considerations debtor nations should insist on their sovereign rights, and social interest groups should take into 
account that the choice of which sectors of society are to bear the burdens entailed by adjustment programmes is a decision of their own governments. Protests ought therefore to be directed against governments and not against the IMF.

\title{
Heading Towards a Second Natural Rubber Agreement?
}

\author{
By Andreas A. B. Hoffmann
}

The International Natural Rubber Agreement of 1979, a commodity price stabilization accord between natural rubber exporting and importing nations, will be renegotiated in 1986. The two groups are divided over the question, whether to include production costs into the formula for calculating the reference price.

Despite diverging views on most issues, producers and consumers agree that chances for concluding a second pact are rather high, compared to futile efforts to achieve working agreements for other raw materials.

It is argued, however, that industrialized rubber importing countries fared better with the present agreement than did the developing exporters, who start to feel the financial burden of maintaining a 380,000 tons buffer stock.

\section{The Chinese Economic Reforms since 1978 with Particular Regard to the Special Econo- mic Zones}

\section{By Martin Klingst}

Since 1978 the People's Republic of China (PRC) has undertaken vast agrarian and industrial reforms. These reforms were distinctly welcomed in capitalist countries as well as harshly criticized by Chinese opponents and various foreign communist movements and parties.

This article, therefore, starts with a question: Is the PRC returning back to capitalism? In the following it deals with the different legal, economic and political aspects of the economic reforms and focuses on the establishment of the so-called "Special Economic Zones", because it was mainly these that gave rise to the above mentioned discussion. Special Economic Zones are zones of free enterprise - mostly export-processing-zones and are to be found all over the world. But especially in the so-called developing countries they provide many favorable conditions for local and foreign investors.

After describing and analyzing the various general aspects of Special Economic Zones and details about the Chinese Special Economic Zones the article ends with the conclusion that the PRC is changing but will nevertheless remain a socialist country, because: 